\title{
LANDAU CAVITIES IN THIRD GENERATION SYNCHROTRON LIGHT SOURCES
}

\author{
M. Georgsson, MAX-lab, Lund University, Lund, Sweden
}

\section{Abstract}

The new $3^{\text {rd }}$ generation synchrotron light sources coming up are designed for a small transverse emittance. But the small beam size gives a low Touschek lifetime and calls for good beam stability. In some storage rings higher harmonic cavities are installed for bunch lengthening to overcome the lifetime limitations. The higher harmonic cavities also induce Landau damping that improves the beam stability; they are therefor often called Landau cavities. A brief introduction to Landau cavities is given. Then the commissioning and use of these cavities at ALS, Bessy, MAX-lab and Super-ACO is described.

\section{INTRODUCTION}

Synchrotron radiation is a tool widely used in science. It is produced by electron accelerators called storage rings. With the high performance of the $3^{\text {rd }}$ generation synchrotron light sources, the scientific results are excellent. It is important to identify and overcome the limitations of a storage ring in order to enhance its performance. This will enable research facilities to withstand competition and serve science for many years to come. Typical limitations are short electron beam lifetimes and induced energy spreads originating from instabilities.

Short beam lifetime is due to the small transverse and longitudinal beam sizes. Increasing the transverse beam size would spoil the small emittance of the electron beam. However, increasing the longitudinal beam size, the bunch length, is an attractive option. This can be realised by adding a passive higher harmonic radio frequency, RF, system.

Longitudinal and transverse feedback systems are often installed to damp coupled bunch instabilities and these feedback systems functions efficiently. A higher harmonic cavity also adds Landau damping. This damping can be so strong that it can be an alternative to the feedback systems in some cases. Higher harmonic cavities are therefore also called Landau cavities.

By installing a Landau cavity system, two performance limitations can be solved. It lengthens the bunches, and with this the lifetime, and it introduces Landau damping that damps instabilities. In storage rings that already have installed feedback systems Landau cavity systems are useful for lifetime improvement.

Here is a short summary of the application of higher harmonic cavities at four SR laboratories. For the theory of the applications presented see reference [1].

\section{MAX II}

The purpose of the Landau cavities in MAX II is twofold: to decrease the energy spread of the beam induced by coupled bunch oscillations and to increase the Touschek lifetime. The initial energy spread was five times larger than the natural and this was seriously affecting the resolution on the undulator beam lines at high harmonics. The lifetime was $12 \mathrm{~h}$ at $200 \mathrm{~mA}$, which is not bad, but if doubled only one injection per day would suffice.

The main RF-system is $500 \mathrm{MHz}$ and $600 \mathrm{kV}$. In this case the third harmonic, $1.5 \mathrm{GHz}$, is a good frequency to construct a cavity at. Five cavities were constructed, but the Q-value and shunt impedance were so good that only four were inserted.

The storage ring was filled with electrons and ramped to $1.5 \mathrm{GeV}$. The Landau cavities were tuned towards their resonance position and very early the beam size decreased and the lifetime was doubled. See fig. 1. It is not necessary to reach the zero-slope condition to achieve a strong effect. More figures are found in [2].

Since 1997 MAX II is routinely operated with the Landau cavities. They are lengthening and stabilising the bunches. The performance enhancement of the undulators is significant as is seen in fig 2 that shows one of the high harmonic undulator peaks.

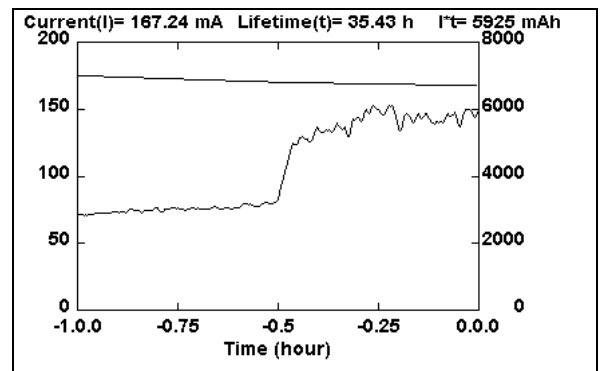

Figure 1: Lifetime increase in MAX II.

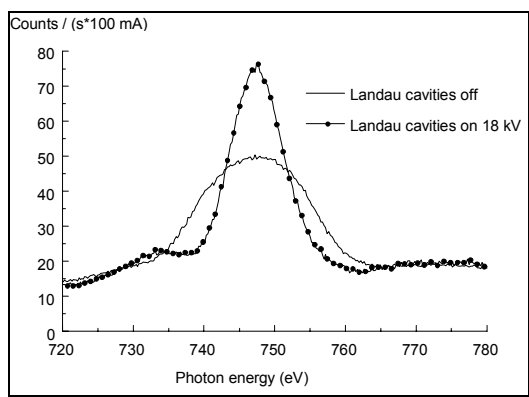

Figure 2: The effect of the Landau cavities on a high harmonic of undulator 411 at MAX II. 


\section{ADVANCED LIGHT SOURCE}

ALS is a $1.5-1.9 \mathrm{GeV}$ electron storage ring. The circumference is $196 \mathrm{~m}$ and the rotational frequency 1.5 MHz. Both transverse and longitudinal feedback systems are inserted to handle energy oscillations. With the stable beam, the electron bunch density is very high and the Touschek lifetime low. Therefore it was decided to insert a harmonic cavity system. $1.5 \mathrm{GHz}$ cavities were designed at LBNL and manufactured by LLNL [3, 4]. It was a rounded design with nosecones. They have both very high $\mathrm{Q}$-values and shunt impedance. There is a large difference in the number of cavities necessary, depending on beam current and energy, to get optimum effect. In ALS five cavities are installed although three would suffice at full energy and maximum current.

It was quite tricky to commission the higher harmonic cavity system. To power the cavities was as easy as at MAX II, but ALS is more sensitive for the HOMs in the $1.5 \mathrm{GHz}$ cavities, especially the first longitudinal mode, the TM011-mode, at $\sim 2.35 \mathrm{GHz}$. A lot of time was spent moving the two plungers in each cavity in order to find safe positions where only the fundamental mode would get excited.

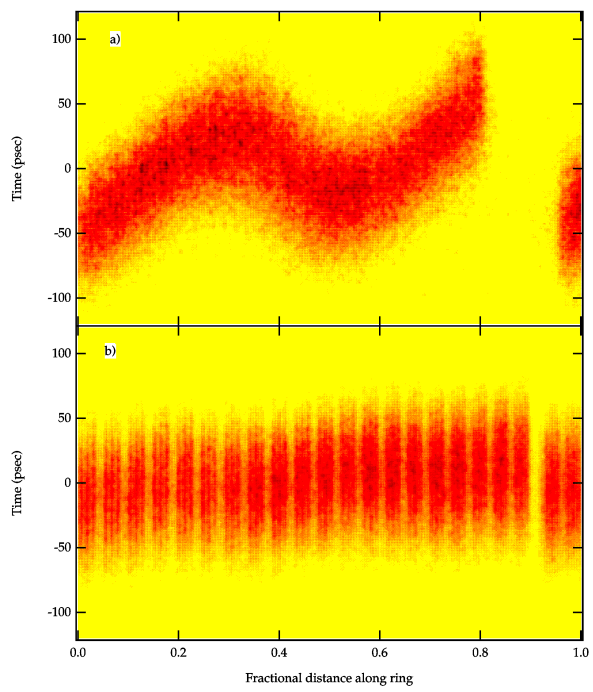

Figure 3: Streak camera images of the longitudinal bunch distribution along the length of the bunch train: (a) 272 bunches: and (b) 320 bunches.

The reason for the more easily excited HOMs in the ALS cavities is that the storage ring is physically larger. With this, the rotational frequency is $1.5 \mathrm{MHz}$ compared to the $3.3 \mathrm{MHz}$ in MAX II, and the risk for hitting a HOM is more than doubled.

Another problem was that the feedback systems were not as effective as usual. ALS operates with a gap in the bunch train. The bunches before and after this gap have different synchronous phases than the other bunches. This is called phase transients and the effect is larger when the gap is increased. See fig 3. The problems with the feedback systems were fixed by changes in the hardware. Later a hardware fault was discovered and corrected. Now the HHC and feedback system co-operate smoothly [5].

The cavities are now operated routinely. The present status is a factor of 2 bunch lengthening with an even fill pattern. At 3\% coupling, the lifetime is 9 hours at $400 \mathrm{~mA}$.

\section{BESSY II}

Bessy II is even larger than ALS and operates normally at $1.7 \mathrm{GeV}$. In the storage ring both transverse and longitudinal feedback systems are installed. The similarity indicated that the commissioning could be as troublesome as at ALS. To decrease the foreseen problems the design of the cavity tuners was modified. Instead of using two identical plungers as at ALS one was placed off-centred for better differential tuning. Four $1.5 \mathrm{GHz}$ cavities were installed into the storage ring.

Commissioning started by careful tuning of the cavities. The cavities were equipped with two diagnostic loops, with these in situ $\mathrm{S}_{12}$ measurements were done. The TM011 could safely be tuned away between two rotation harmonics. The fundamental mode was tuned in the same way to bunch lengthening. See fig 4.

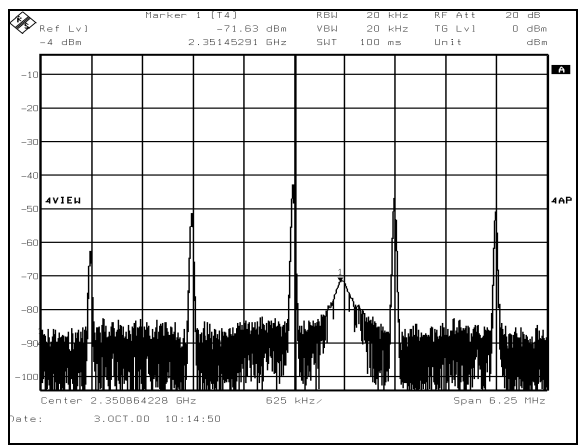

Figure 4: In situ $\mathrm{S}_{12}$ measurement showing the TM011 mode of a Landau cavity tuned in between two rotational bands. Bessy

The cavity tunings were kept fixed during the fill of the storage ring. As the current increased the cavity voltages increased and the bunches were lengthened. This is seen in figs 5 a $\&$ b. The Landau cavities are presently used in routine operation [6].
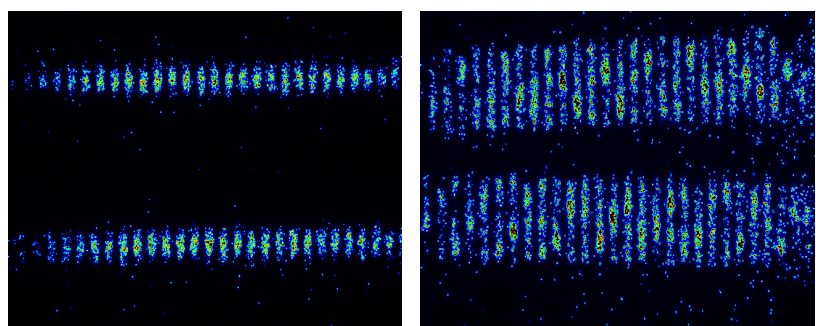

Figure 5: a. $121 \mathrm{~mA}$ Landau cavities tuned to parking position, feedback on. $200 \mathrm{~ns} \mathrm{x}$-axis and $706 \mathrm{ps} \mathrm{y}$-axis. $125 \mathrm{MHz}$ synchroscan mode. b. $220 \mathrm{~mA} 1 \mathrm{MV}$ in the main cavity and totally $140 \mathrm{kV}$ in the Landau cavities, feedback on. $200 \mathrm{~ns} \mathrm{x}$-axis and $1152 \mathrm{ps}$ y-axis. $125 \mathrm{MHz}$ synchroscan mode 


\section{SUPER-ACO}

Normally Super-ACO has longitudinal multibunch instabilities as is seen in figs 6 a \& b. These instabilities were damped totally as seen on both the spectrum analyser and the streak camera, as seen in figs 7 a \& b.

When tuning the cavity towards the resonance, the effective bunch length decreased, the synchrotron frequency decreased and disappeared, and the spectrum became purer with only very small sidebands. Then, the effective length increased and 2 small side bands appear. Meanwhile the energy dispersion continued to decrease and we reached the minimum for about $34 \mathrm{kV}$, which correspond to the zero-slope. The minimum energy spread, $\sigma_{\varepsilon}=6 \cdot 10^{-4}$ for $200 \mathrm{~mA}$ and $\sigma_{\varepsilon}=6.5 \cdot 10^{-4}$ for $400 \mathrm{~mA}$, is just slightly higher than the natural value at zero current, $\sigma_{\varepsilon}=5.3 \cdot 10^{-4}$. The coupled bunch instabilities were almost completely cancelled. The users noticed an increase in brilliance, as well as an improvement of the stability of the beam. [7]

This configuration is not used routinely for the moment because the lifetime is a bit shorter, $12 \mathrm{~h}$ instead of $13 \mathrm{~h}$, than at the usual point with coupled bunch instabilities which give a larger effective bunch length.
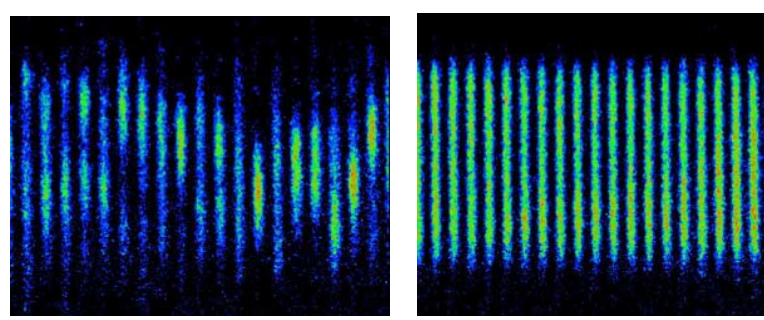

Figure 6: a. Streak camera picture of normal operation in Super-ACO. $\mathrm{I}=200 \mathrm{~mA}$ in 24 bunches. b. $\mathrm{I}=400 \mathrm{~mA}$ in 24 bunches. Elongated and stabilised bunch train in Super-ACO
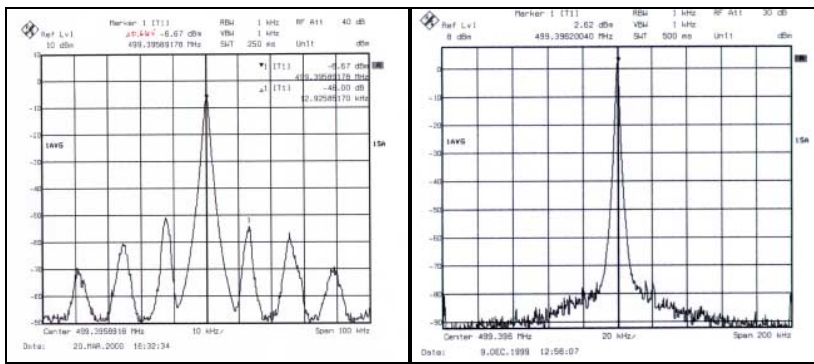

Figure 7: a. Synchrotron tunes with the $500 \mathrm{MHz}$ cavity detuned. b. The synchrotron tunes has disappeared with $34 \mathrm{kV}$ in the $500 \mathrm{MHz}$ cavity.

\section{CONCLUSIONS}

Adding a higher harmonic cavity system for bunch lengthening in a low to medium energy synchrotron light source can lead to a significant enhancement of the accelerators performance.

The stable bunch lengthening effect is clear with four cases presented here. The corresponding lifetime increases depend on the initial beam parameters. In both
MAX II and ALS the users experience a significant enhancement in beam lifetime. Also in Bessy II, the beam lifetime is improved and the effect will be even stronger as the vacuum system gets fully commissioned. However, the users at Super-ACO experienced a $10 \%$ lifetime decrease. The reason for this is the instabilities present in the initial condition effectively lengthened the bunches even more, with the increased energy spread that followed.

The coupled bunch oscillations are damped in both MAX II and Super-ACO, to the extent that the machines are considered to be stable. This shows the effectiveness of Landau damping. This was not studied at ALS or Bessy II due to their installed feedback systems.

At ALS and Bessy II the commissioning was more complicated then at MAX II despite the similarities of the systems. One reason for this was the size of the accelerators, and this in two ways. Firstly, the revolution frequency is $1.5 \mathrm{MHz}$ for $\mathrm{ALS}$ and $1.25 \mathrm{MHz}$ for Bessy II. This is less than half the value for MAX II, 3.3 MHz. This made it more complicated to avoid higher order modes in the RF-cavities. Secondly the radiation damping is stronger in MAX II, due to the strong bending-magnet fields. This also makes it harder for instabilities to grow. The other reason for commissioning difficulties is that ALS and Bessy II operate longitudinal and transversal feedback systems and special care is necessary in order to operate these systems together with the harmonic cavities.

Adding passive harmonic cavities have highly enhanced the performance of these $3^{\text {rd }}$ generation synchrotron light sources, to the benefit of their synchrotron radiation user community.

\section{REFERENCES}

[1] Georgsson, M. "Higher harmonic cavities at third generation synchrotron light sources", $\mathrm{Ph} . \mathrm{D}$. Thesis, ISBN 91-7874-131-9, 2001

[2] Georgsson, M., Å. Andersson, M. Eriksson, "Landau cavities in MAX II", Nucl. Instr. and Meth. A 416 pp. 465-474, 1998

[3] Rimmer, R.A., K. Baptiste, J. Byrd, T. Hendersen, C.C. Lo, D. Plate, "A third harmonic cavity for the advanced light source", EPAC'98, Stockholm, pp. 1808-1810, 1998

[4] Byrd, J.M., et. Al., "Design of a higher harmonic RF system for the Advanced Light Source", Nucl. Instr. and Meth. A 439 pp. 15-25, 2000

[5] Byrd, J.M., et. Al., "Commissioning of a higher harmonic RF system for the Advanced Light Source", Nucl. Instr. and Meth. A 455 pp. 273-284, 2000

[6] Georgsson, M., W. Anders, D. Krämer and J. M. Byrd, "Design and Commissioning of 3 rd harmonic Cavities at BESSY II", Nucl. Instr. and Meth. A, accepted for publication

[7] Georgsson, M. et. al., "Using Super-ACO's fifth harmonic cavity as a passive Landau cavity", EPAC'00 Vienna, pp 1960-1962. 